\title{
Identification of Vehicle by Number Plate Recognition Using Genetic Algorithm
}

\author{
Samira Nigrel ${ }^{1}$, Akash Ashokan ${ }^{2}$, Bidisha Barua $^{3}$, Slesha Patil $^{4}$, Vaibhavi Patil ${ }^{5}$ \\ Lecturer, Information Technology, Atharva College of Engineering, Mumbai, India ${ }^{1}$
}

Student, Information Technology, Atharva College of Engineering, Mumbai, India ${ }^{2,3,4,5}$

\begin{abstract}
Design of an algorithm for vehicle identification by recognizing the number plate is presented. Automatic Vehicle Identification (AVI) has many applications in traffic systems (highway electronic toll collection, red light violation enforcement, border and customs checkpoints, etc.).The proposed algorithm consists of three major parts: Number plate recognition, vehicle size detection and recognition of plate characters. For extracting the plate region, edge detection algorithms and smearing algorithms are used. In segmentation part, smearing algorithms, filtering and some morphological algorithms are used. And finally genetic algorithm is used for recognition of plate characters. For vehicle size detection blob detection method is used and for theft detection number plates are matched. Experiments have been conducted with images taken from various scenes and conditions and the detection rate is found to be 92.5 $\%$. Experiments have conducted for recognition with LPR images taken at different conditions and the recognition rate is found to be $91 \%$. Detection time is linear function of number of objects in the input image. Potential applications include provisioning of vehicle parking facilities and campus security system for permitting authorized vehicles into the premises.
\end{abstract}

Keywords: Number Plate Recognition, Genetic algorithm, plate region extraction, segmentation, thinning, vertical and horizontal projection.

\section{INTRODUCTION}

Number Plate Recognition is used increasingly. Nowadays for automatic toll collection, maintaining traffic activities and law enforcement. Therefore, control of vehicles is becoming a big problem and much more difficult to solve. Number plate vehicle identification systems are used for the purpose of effective control [2]. Number plate remains the principal vehicle identifier despite the fact that it can be deliberately altered in fraud situations or replaced (e.g., with a stolen plate). Therefore, ITS rely heavily on robust number plate recognition. A number plate is used to identify each vehicle uniquely, which states a legal license to participate in the public traffic. Vehicles all over the world should have its own unique number plate - mounted onto its body (at least at the back side). A vehicle without a properly mounted and well noticeable number plate should not run on the roads. To process, arrange or evaluate data everyone thinks about using computers. If the data is already in the computer most of these tasks are rather easy to be carried out. It is needless to say that the plate number is the most important identification data a computer system should treat when dealing with vehicles. Our aim is to identify vehicle by number plate recognition using Genetic algorithm. The objectives for the proposed method are firstly the recognition of number plate to identify the vehicle. For this, we use the method of blob detection and thresholding. Then, the second step is feature based matching of characters. For this Genetic Algorithm is used. Additional features included in this proposed method would be alarm for violation of traffic rules or theft and also the determination of size of the vehicle (small/medium/large).

If implemented, the proposed method has various useful scopes such as it can be used for security purposes in restricted areas, can be used at traffic signals for traffic defaulters, also it can be used by Authorities for stolen vehicles.

Automatic vehicle identification systems are used for the purpose of effective control. Automatic Number Plate Identification (ANPI) is a form of automatic vehicle identification. This technique of processing an image is used to identify vehicles by only their number plates. Real time NPR plays a major role in automatic monitoring of traffic rules and maintaining law enforcement on public roads. Since there is a unique number plate for every vehicle, there is no need of any external cards, tags or transmitters for recognizing a vehicle, only the number plate is sufficient. In this paper, the proposed algorithm is based on extraction of plate region, segmentation of plate characters and recognition of characters. Extraction of plate is a difficult task. The various difficulties faced during the extraction of plate can be due to the reason that number plates occupy a very small portion of the entire image, and there is a large variety in fonts, colours and styles in our country [1]. Also, in most cases, the detecting is performed without prior knowledge of the number plate's location in the image. The common factors that would affect the efficiency of extraction would be low resolution of image, distorted characters, shadows or reflection, unclean plate, low illumination, fuzzy images, etc [1]. Additionally, real time extraction of number plate is a complex procedure as it requires high speed as well as accuracy.

So many researches of car identification have been approached by car license plate extracting and recognition, some of the related work is as follows. Johnson proposed automatic number-plate recognition using optical character 
recognition techniques [3]. Johnson and Bird proposed areas covering those regions. A blob is a part of a digital knowledge-guided boundary following and template image in which some attributes are fixed or change within matching for automatic vehicle identification [4]. Fahmy a predefined range of values; all the points in a blob can be proposed bidirectional associative memories (BAM) used in some sense to be alike each other.

neural network for number plate reading [5]. It's B. LOCALIZATION \& EXTRACTION OF NUMBER appropriate for small numbers of patterns. Nijhuis, Ter PLATE:

Brugge, Helmholf J.P.W. Pluim, L. Spaanenburg, R.S. The extraction of plate region is the first step of the Venema and M.A.Westenberg proposed fuzzy logic and algorithm. Image captured from the camera is first neural networks for car number plate recognition. converted to the binary image which exists of only 1 's and The paper is organized as follows: Section III contains 0's (only black and white) [2] by using the method of proposed method, Section IV contains Experimental thresholding. In this method, the pixel values of 0 (black) Results, and conclusion and References.

\section{Proposed Method}

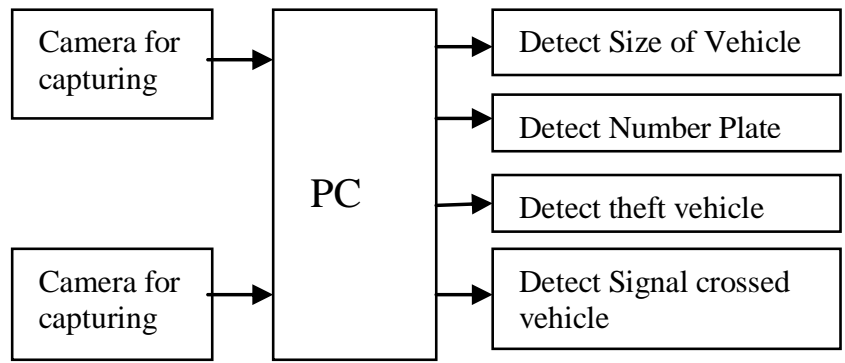

Fig. 1. Structure of the NPR System

Here two cameras are used one for vehicle and one for number plate capturing. We will have database of registered number plate, Character database (0-9 and A to $\mathrm{Z}$ ).When vehicle comes, its size will be detected through blob detection. Then Number plate will be detected using Genetic Algorithm and verified if is registered or not. Then will have red, green and blue indicators simulation. If red signal is active and vehicle is passed then its number plate, fine and date, time will be stored in database. If someone's vehicle is stolen then he will enter theft on signal in database. If that vehicle comes and is theft signal on the buzzer signal will be activated.

Traffic images are captured and it is compensated for noise and lighting differences. Edge and gradient techniques are in use to extract fore ground details. Number of object areas in the image is found out by counting the gray level transitions. Windows equal to number of object areas are shown with left, right, top and bottom line. Based on the geometrical details of the detected window such as height, width and aspect ratio, objects regions are differentiated into small, medium and heavy vehicles [1]. Then, number plate is localized and extracted by pixel processing techniques. GA is employed at the second level to control and map the character pixels into the window panes. Finally, a features based matching is employed for character recognition.

The implementation can be carried out in four stages as follows:
A. Blob Detection, [2]
B. Localization and Extraction of Number Plate, [1]
C. Segmentation, [2] \&
D. Character Recognition using Genetic Algorithm [1].
A. BLOB DETECTION:

In this detecting regions in a digital image that vary in attributes such as brightness or colour, are compared to are given for all pixels in the input image with light factor less than threshold value. Also the pixel value of 1 (white) is given for all other pixels [2]. The processing of the binarized image is carried out with the help of some methods. To obtain the plate area, smearing algorithm is used initially. The method for the extraction of text regions on a mixed image is called as smearing. The image is refined along vertical and horizontal runs (scan-lines) using the smearing algorithm. If the number of white pixels is less than the predefined threshold or greater than any other predefined threshold, white pixels are made black. Threshold values are selected as 10 and 100 for both horizontal and vertical smearing in this system. If number of 'white' pixels $<10$; pixels become 'black'

Else; no change

If number of 'white' pixels > 100; pixels become 'black'

Else; no change [2]

Once smearing is completed, dilation which is a morphological operation, is employed on the image for distinguishing the plate region. However, there can be more than one candidate region for plate location [2]. Some criteria tests are applied to the image by smearing and filtering operation to find the exact region and eliminate the other regions. After this stage the processed image is as shown in Figure 2(a) and image involving only plate is shown in Figure 2(b).

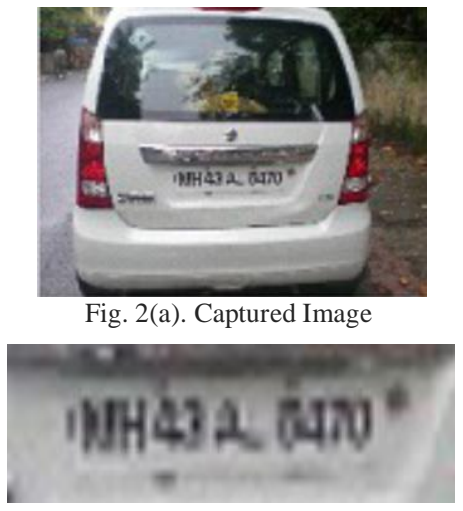

Fig. 2(b). Extracted Number Plate

\section{SEGMENTATION:}

Number plate is segmented into its components obtaining the characters one by one in the segmentation procedure. In the first step, image is filtered for improving the attributes of the image and eliminating the noises and unwanted spots. Then dilation operation is applied to the image for distinguishing the characters from each other which is required if the characters are close to each other. 
Horizontal and vertical smearing are applied for finding the character regions after segmentation and dilation operations are carried out [2]. The next step is to separate the plate characters. It is carried out by detecting the starting and end points of characters in horizontal direction. The individual characters separated from the plate are as follows in Figure 3.

\section{$\mathrm{MHO4}$}

Fig. 3. Segmented Characters

\section{CHARACTER RECOGNITION USING GENETIC ALGORITHM:}

GA is employed for recognition of character at the second level. String for character recognition consists of 6 bits shown in Figure 4. The first bit is to decide if the captured character is a number or an alphabet. The next bit is to decide if the captured character matches more than one. The rest of the 4 bits represent mapping of 4 window panes of digital board.

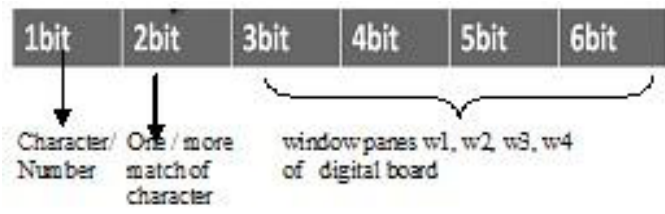

Fig. 4. String for Character Recognition

\section{ALGORITHM:}

The main objective of the algorithm is to detect the number plates of the various vehicles automatically. the image of a vehicle captured by a camera is considered as the input of the system. The image which is captured is taken from 3-4 meters away, is refined with the help of the number plate extractor which gives its output to the segmentation part. Segmentation part distinguishes the characters one by one. And finally recognition part recognizes the characters which give its result as the plate number [1].

Read jpeg,file

Extract fore ground object details;

Decode a string for detection;

\{ struct of GA for detect

generate initial population;

fitness evolution;

reproduction;

cross over;

mutation \};

Train for more traffic images;

Localize and extract Number plate

$\mathrm{cx}=\operatorname{dim}[0] / 2$;

$\mathrm{cy}=\operatorname{dim}[1] / 2$;

string is decoded for recognizing characters

\{ struct of GA for character recognize

bit=intarr(7,2, windows +1$)$;

A DBF is designed for character recognition

character $[1, \mathrm{i}]=\operatorname{total}(\mathrm{w} 1)$;

character $[2, \mathrm{i}]=\operatorname{total}(\mathrm{w} 2$; $\operatorname{character}[3, \mathrm{i}]=\operatorname{total}(\mathrm{w} 3)$;

Character $[4, \mathrm{i}]=\operatorname{total}(\mathrm{w} 4)$;

\{generate initial population;

fitness evolution;

reproduction;

cross over

mutation

Use for more images containing characters [2].

\section{III.EXPERIMENTAL RESULTS}

Experiments have been performed to test the proposed system and to measure the accuracy of the system. The system is designed in Matlab for recognition of number plates. The images for the input to the system are colored images with the size of $1200 \times 1600$. The test images were taken under various illumination conditions. The results of the tests thus performed are given by the Table below:

TABLE I

RESULTS OF THE TESTS

\begin{tabular}{|l|l|l|}
\hline $\begin{array}{l}\text { Units of NPR } \\
\text { system }\end{array}$ & $\begin{array}{l}\text { Number of } \\
\text { Accuracy }\end{array}$ & $\begin{array}{l}\text { Percentage of } \\
\text { Accuracy }\end{array}$ \\
\hline $\begin{array}{l}\text { Extraction of } \\
\text { Plate Region }\end{array}$ & $166 / 170$ & $97.6 \%$ \\
\hline Segmentation & $163 / 170$ & $96 \%$ \\
\hline $\begin{array}{l}\text { Recognition of } \\
\text { Characters }\end{array}$ & $168 / 170$ & $98.8 \%$ \\
\hline
\end{tabular}

It is shown that accuracy for the extraction of plate region is $97.6 \%, 96 \%$ for the segmentation of the characters and $98.8 \%$ is the percentage of accuracy of the recognition unit. The overall system performance can be defined as the product of all units accuracy rates (Extraction of plate region, segmentation of characters and recognition of characters). Recognition Rate of LPR System $=$ pi (Percentages of Accuracy)

For vehicle detection in [6], a data base of pictures with their locations and class of objects were manually determined. This algorithm for vehicle identification by plate recognition uses GA. The new algorithm is tested on road traffic images captured from CCTV cameras in national highways in India also. Traffic images may consist of variety of vehicles like bus, truck, scooter, car, bike, motor cycle etc. This algorithm is implemented on Intel Core to Duo Processor in Windows XP using RSI's IDL platform. Experiments are carried out with images from NPR images. Edge based segmentation is adopted to detect object regions and they are captured in different windows using GA. If more than one object is present in a single window, overlapping region is detected and detached. Experiments are repeated for detection with 8 bit string using a separate bottom line for every window. Experimental results are better for edge images obtained 
by gradient methods than threshold methods. To detect vehicle categories, Shape information is employed. Shape features like height, width, aspect ratio of all the road vehicles are computed and stored in a separate data base. Experiments have conducted with over 100 images taken from a variety of conditions.

In [7], a template with two rectangles was used to detect the number plate. The positioning, size, positions of the outer and inner rectangles, orientations were controlled by GA. Character recognition, neural networks and template matching was united. GA program for recognition of character is written and it is applied over the character's edge image to calculate its feature vector using the DB. Detection time is a linear function of number of objects in the input image.

\section{ConCluSION}

In this paper, we have presented a simple but efficient method of extracting the number plate of various vehicles. The proposed method consists of four major stages which include RGB to gray-scale conversion, image binarization and filtration, analysis and dilation, and extracting the accurate location of the number plate. The algorithm presented in this paper could detect the vehicles and recognize the characters in the number plate quickly with good accuracy. Various experiments have been conducted to test the efficiency, like obtaining a number of images varying in illumination and weather conditions, and achieved satisfactory results. The proposed method can be modified and can be applied to any system with its set of requirements.

\section{ACKNOWLEDGMENT}

It gives us great pleasure in presenting this project report titled:"Identification of Vehicle by Number Plate Recognition Using Genetic Algorithm".

On this momentous occasion, we wish to express our immense gratitude to the range of people who provided invaluable support in the completion of this project. Their guidance and encouragement has helped in making this project a great success.

We express our gratitude to our project guide Prof Samira Nigrel, who provided us with all the guidance and encouragement and making the lab available to us at any time. We also would like to deeply express our sincere gratitude to Project coordinators.

We are eager and glad to express our gratitude to the Head of the Information Technology Dept. Prof Neelima Pathak, for her approval of this project. We are also thankful to her for providing us the needed assistance, detailed suggestions and also encouragement to do the project.

We would like to deeply express our sincere gratitude to our respected principal Prof. Dr. Shrikant Kallurkar and the management of Atharva College of Engineering for providing such an ideal atmosphere to build up this project with well equipped library with all the utmost necessary reference materials and up to date IT Laboratories

We are extremely thankful to all staff and the management of the college for providing us all the facilities and resources required.

\section{REFERENCES}

[1] P. Vijayalakshmi, M. Sumathi” Design of Algorithm for Vehicle Identification by Number Plate Recognition" IEEE- Fourth International Conference on Advanced Computing, ICoAC 2012, MIT, Anna University, Chennai. December 13-15, 2012

[2] Serkan Ozbay, and Ergun Ercelebi, "Automatic Vehicle Identification by Plate Recognition", World Academy of Science, Engineering and Technology International Journal of Electrical, Robotics, Electronics and Communications Engineering Vol:1 No:9, 2007

[3] R.A. Lotufo, A.D. Morgan, and AS. Johnson, 1990, "Automatic Number-Plate Recognition," Proceedings of the IEE Colloquium on Image analysis for Transport Applications, V01.035, pp.6/1-6/6, February 16, 1990.

[4] A.S. Johnson, B.M. Bird, 1990, "Number-plateMatching for Automatic Vehicle Identification," IEE Colloquium on Electronic Image and Image Processing in Security and Forensic, Aprl, 1990.

[5] M.M.M. Fahmy, 1994, "Automatic Number-plate Recognition : Neural Network Approach," Proceedings of VNIS'94 Vehicle Navigation and Information System Conference, 31 Aug-2 Sept, 1994

[6] Mengjie Zhang, Victor Ciesielski, Genetic Programming for Multiple Class Object Detection, Springer Berlin / Heidelberg, ISBN No. 978-3-540-66822-0, 2007.

[7] Stephen Karungaru, Minoru Fukumi, Norio Akamatsu, "Detection and Recognition of Vehicle NumberPlates Using Template Matching, Genetic Algorithms And Neural Networks", International Journal of Innovative Computing, Information and control, ISSN 1349-4198 Volume 5, Number 7, pp. 1975-1985, July 2009.

[8] Y. Cao, A. Renfrew, P. Cook, "Vehicle Motion Analysis Based on a Monocular Vision System", Road Transport Information and Control- RTIC 2008 and ITS United kingdom Members' conference, pp.1-6, 20- 22.

[9] A. Haselhoff, S. Schauland, A. Kummert, "A Signal Theoretic Approach to Measure the Influence of Image Resolution for Appearance- based Vehicle Detection", Intelligent Vehicles Symposium, IEEE, June 2008. pp: 822 - 827

10] Y. Chan, S. Huang, L. Fu, P. Hsiao, "Vehicle Detection under Various Lighting Conditions by Incorporating Particle Filter", Intelligent Transportation Systems Conference, 2007, pp.534-539.

[11] S. M. Abdel- gaied, "Employing Genetic Algorithms for qualitative Shapes Detection", ICGST-GVIP, December 2008, ISSN 1687 398X, Volume (8), Issue (IV).pp:18-25

[12] O.L. Junior, U. Nunes, "Improving the Generalization Properties of Neural Networks: an Application to Vehicle Detection", Intelligent Transportation Systems, , 11 th International IEEE Conference on, 2008, pp.310-315, $12-15$

13] ozbay, and Ergun Ercelebi, Serkan, "Automatic Vehicle Identification by Plate Recognition", A World Academy of Science, Engineering and Technology, pp:222-225, 2005 